\title{
Decomposition of Electromagnetic Interferences in the Time-Domain
}

\author{
Marco A. Azpúrua, Member, IEEE, Marc Pous, and Ferran Silva, Member, IEEE
}

\begin{abstract}
Electromagnetic interferences are potentially very complex signals formed by the superposition of transient (broadband) and continuous wave (narrowband) components with significant randomness in both amplitude and phase. Decomposing the electromagnetic interference measured in the time domain into a set of intrinsic mode functions is useful to gain insights of the process that generates the interference. Evaluating the intrinsic mode functions contributes to improving the measurement capabilities of the time-domain electromagnetic emissions measurement systems based on the general-purpose oscilloscopes. In this paper, a combination of techniques that includes empirical mode decomposition and transient mode decomposition is used to separate the main components of complex electromagnetic disturbances. This approach requires no prior information on the spectral content of the measured EMI and it does not perform a domain transformation. Examples of electromagnetic interference decomposition verify the effectiveness and the accuracy of the proposed approach. Finally, a discussion on the advantages, practical applications, limitations, and drawbacks of the described techniques is addressed.
\end{abstract}

Index Terms-Digital signal processing, electromagnetic compatibility, electromagnetic interference, electromagnetic measurements, time-domain analysis.

\section{INTRODUCTION}

$\mathbf{E}$ LECTROMAGNETIC interferences are complex signals composed of the superposition of continuous-wave, transient, and random disturbances. In consequence, measuring properly and defining specific features of an electromagnetic interference (EMI) are challenging tasks.

In this regard, the conventional approach for the evaluation of the electromagnetic emissions consists in measuring the amplitude spectrum of the disturbance using a frequency sweep receiver and the CISPR standard detectors [1]. Therefore, the current standard measurement procedures for assessing an EMI neglect important time-domain characteristics of the electromagnetic disturbances, such as the repetition rate, the different emission profiles of an EUT as it changes its operation mode, and the impact of transient events [2]. This is particularly relevant for evaluating and predicting the degradation suffered by digital

Manuscript received June 16, 2015; revised November 20, 2015; accepted January 4, 2016. This work was supported in part by the EURAMET IND60EMC research project (the EMRP is jointly funded by the EMRP participating countries within EURAMET and the European Union) and by the Spanish "Ministerio de Economía y Competitividad," under project TEC201348414-C3-3-R.

The authors are with Group of Electromagnetic Compatibility, Department of Electronic Engineering, Polytechnic University of Catalonia, Barcelona 08034, Spain (e-mail: marco.azpurua@upc.edu; marc.pous@upc.edu; ferran.silva@ upc.edu).

Color versions of one or more of the figures in this paper are available online at http://ieeexplore.ieee.org.

Digital Object Identifier 10.1109/TEMC.2016.2518302 communication systems due to the influence of a transient EMI [2]. Consequently, in some cases, the standardized methods of EMI measurement and evaluation are not completely suitable, insufficient or might require an enormous amount of time to provide reliable measurement results [3], [4].

Currently, the fast Fourier transform-based EMI test receivers and real-time spectrum analyzers have time-scan measurement capabilities that overcome many of the limitations of the stepped frequency scan in the EMI receivers. However, the bandwidth of their intermediate frequency filter and the tradeoff between the time domain and the frequency-domain resolutions [5] are their main constraints for measuring simultaneously broadband and narrowband interferences on the whole frequency range. Recently, advances in an EMI postprocessing and the enhanced capabilities of digital oscilloscopes allowed to implement entirely time-domain EMI (TDEMI) measurement systems. Those TDEMI systems provide accurate, full spectrum, multichannel, time saving, and cost-effective EMI measurements [6].

Nonetheless, estimating the spectral content of an EMI is only one side of a multifaceted problem. For example, transient, intermittent, or event triggered (i.e., by changing operation modes) disturbances could be unnoticed by conventional frequency sweep EMI measurements because the unsynchronized occurrence of the EMI and its short duration. Previous research has addressed some of those problems using timedomain EMI analysis. In particular, Alban et al. [7] used an ad hoc oscilloscope-based TDEMI measurement system for developing a statistical model for the broadband noise in computing platforms. In the field of power electronics, time-domain EMI measurements have also been used for providing timefrequency-energy distributions by means of the wavelet analysis used for emissions mitigation in chaotic converters [8]. More recently, statistical approaches to the evaluation of EMI measured in the time-domain have been used to predict the degradation caused by transient disturbances on digital communication systems [2], [9] and to model the distribution of peak measurements using extreme value theory [10].

In that sense, exploring new processing approaches and techniques is fundamental to enhance the performance of such TDEMI systems. In particular, this paper presents an entirely time-domain method that comprises techniques and algorithms for signal decomposition that offer further possibilities to EMI analysis. This method is based on the empirical mode decomposition (EMD) [11] and includes a preliminary stage of transient EMI separation that improves EMD performance.

The decomposition of an EMI in the time-domain seeks to decompose the main components of the measured signal 
without applying a domain transformation. Thus, the transient and continuous-wave modes identified usually have a clearer physical meaning and make it possible to apply specific digital processing techniques for improving the overall measurement result in terms of dynamic range and accuracy.

The structure of the paper is as follows: Section II introduces the EMD and discusses its suitability for analyzing EMI. Then, Section III presents an algorithm for separating the transient pulses from the measured EMI. Through the application examples (Section IV), it will be verified how the aforementioned techniques can also be used to effectively improve the measurement results when transformed to the frequency domain.

Finally, it is important to state that the developed algorithms were mostly implemented in MATLAB code. Therefore, the nomenclature used in the algorithms written as pseudocode is influenced by the MATLAB syntax and functions.

\section{EMPIRICAL MODE DECOMPOSITION}

The EMD is a method developed for analyzing nonlinear and nonstationary signals. It was introduced by Huang et al. in 1998 [12]. EMD has been successfully used to process biological signals [13] and images [14] among other applications. The main capability of the EMD is to decompose complicated datasets into a finite, and often small, number of components called intrinsic mode functions (IMF) that admit well-behaved Hilbert transforms.

Thus far, EMD has not been used to decompose EMI. However, as time-domain techniques gain more acceptance in the EMI assessment, EMD could provide insights to the understanding of the processes generating the disturbance emissions. Next, the EMD algorithm will be explained in terms of its applications for the EMI decomposition.

\section{A. Overview of the EMD algorithm}

The foundation of the EMD is to study heuristically the local oscillations of the signal. This is achieved by looking at the evolution of a signal between two consecutive local extrema in order to define a high-frequency function containing its details, features, and shape characteristics. This detailed oscillation is contained between two minima and includes, necessarily, a local maximum peak value. On the other hand, the complementary low-frequency part (local trend) of the signal is called residual $r(t)$. Then, the aforementioned process is iteratively repeated on the successive residuals for all the oscillations composing the entire signal through a process called "shifting," until all IMF have been found [15]. If the residual is monotonic, it means there are no more IMF to be decomposed, then the shifting algorithm stops the iteration and the EMD is complete. Fig. 1 shows a simplified flowchart for the shifting algorithm of the EMD method.

Then, for a given signal in the time domain $x(t)$, the EMD constructs an equivalent representation in terms of the linear combination of the IMF $c_{n}(t)$, and the residual, as

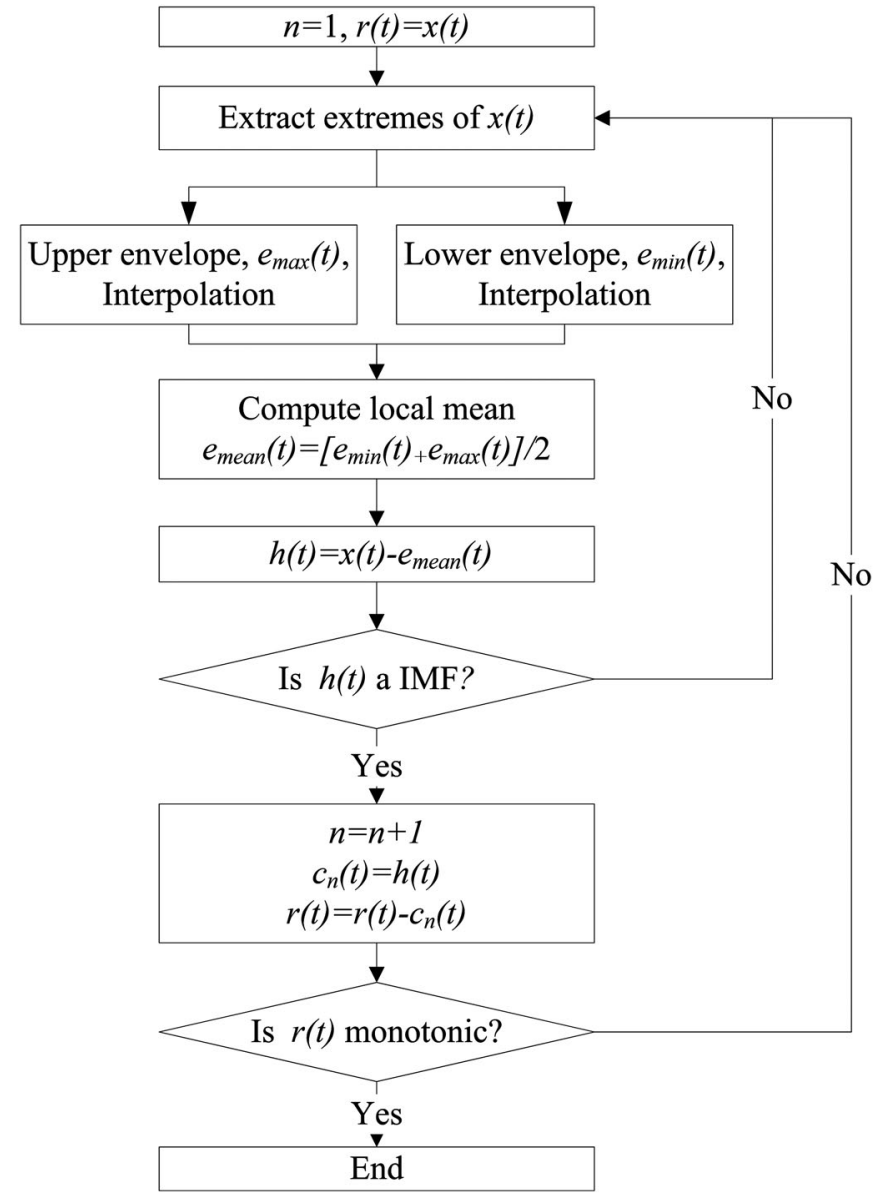

Fig. 1. Flowchart for the "shifting" algorithm of the EMD method. Note: other stopping criteria for the iteration process have also been used [16].

expressed by

$$
x(t)=\sum_{n=1}^{N} c_{n}(t)+r(t)
$$

where each mode is constrained to be zero mean and amplitude/frequency modulation waveforms. A complete explanation of the properties of the EMD is provided in [12].

\section{B. EMD of EMIs}

Using EMD for analyzing EMI is challenging because of the "mode mixing" phenomena. Mode mixing refers to the presence of oscillations of very disparate amplitude in an IMF or the presence of very similar oscillations in different IMF. Mode mixing often take place when dealing with datasets containing intermittent pulses and noise [17], as usually occurs with EMI.

Additionally, the direct application of the EMD algorithm on measured EMI signals with transient components requires enormous amount of time to reach the stop criteria, because the algorithms attempt to overdecompose the transient pulses, leading to IMF with no clear meaning. 
To overcome these drawbacks, it is proposed to separate the transient pulses from the measured EMI in the time domain, as will be explained in the next section. In this case, the transient signal extracted from the EMI is considered to be the first IMF. Then, a variant of the EMD called ensemble empirical mode decomposition (EEMD) is applied on the first residual component of the EMI. EEMD performs the EMD over an ensemble of the signal and additive white Gaussian noise (AWGN). The addition of the AWGN aids solving the mode mixing problem by populating the whole time-frequency space to take advantage of the dyadic filter bank behavior of the EMD [18]. An implementation of the EEMD algorithm has been developed and made available by [19].

\section{TRansient Mode Decomposition}

Before applying EEMD on the measured EMI, the transient pulses shall be separated from the continuous EMI. This is achieved through the automatic recognition of the transients as high amplitude and short-duration signals. By identifying the transient mode within the EMI, it is also possible to estimate its pulse repetition frequency. In order to do so, several processing steps are required due to the potential complexity of the measured EMI. The transient mode decomposition (TMD) algorithm has been implemented in three stages: 1) envelope detection, 2) identification of the transient events and, 3) estimation of the pulse repetition frequency. In the following sections, this algorithm will be explained.

\section{A. Envelope Detection}

First, the measured EMI in the time domain $x(t)$ is processed to obtain its analytic signal $x_{a}(t)$, that is,

$$
x_{a}(t)=A(t) e^{j \phi(t)}=x(t)+j H\{x\}(t)
$$

where $A(t)$ is the instantaneous amplitude and $H\{x\}(t)$ is the Hilbert Transform of $x(t)$, which is given by [20]

$$
H\{x\}(t)=\frac{1}{\pi} \int_{-\infty}^{\infty} \frac{x(\tau)}{t-\tau} d \tau .
$$

The instantaneous amplitude allows a better identification of the features of the signal, such as peaks. Next, the algorithm builds a vector with the local maxima found in $A(t), x_{\text {peak }}$, and a vector containing their corresponding time of occurrence $t_{\text {peak }}$. Considering EMI measurements that are intrinsically noisy, the algorithm uses functions for robust peak detection in the presence of random noise that include prominence criteria between adjacent peaks [21].

In general, the elements of $x_{\text {peak }}$ are not uniformly distributed in time. Therefore, the robust envelope signal $x_{\text {env }}(t)$ is obtained by interpolating $x_{\text {peak }}$ within the measured time interval and then $x_{\text {peak }}$ is resampled at the same rate. The specific interpolation method implemented in this part of the algorithm was cubic interpolating splines since it is fast, efficient, and stable [22]. However, other shape preserving interpolation methods can be used. Finally, $x_{\mathrm{env}}(t)$ is scaled to the amplitude of $x(t)$. The simplified algorithm for envelope detection is described as pseudocode.

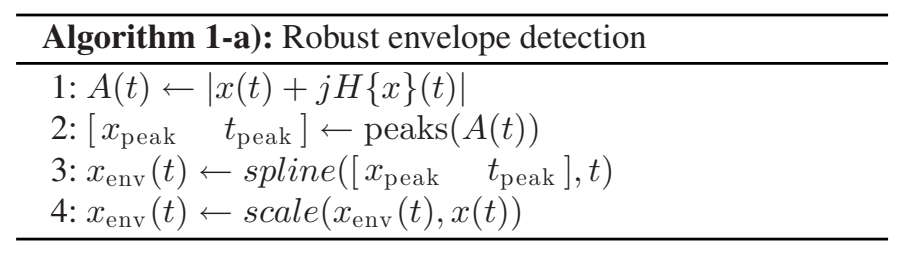

\section{B. Identification of Transient Events}

TMD second step consists of identifying the start and stop instants of the transient events. For accomplishing this, it is required to define a threshold level $\delta$ used as a virtual trigger point for analyzing the transients in $x_{\mathrm{env}}(t)$. However, considering the variability of the peaks, $\delta$ must be empirically estimated to provide sufficient sensitivity to the algorithm.

In this regard, $\delta$ is calculated considering the ranges of amplitude of the prominent peaks of the envelope signal. Then, an observation window $x_{w}$ is defined as a subinterval of $x_{\mathrm{env}}(t)$ extracted to evaluate locally the behavior of the envelope signal in order to decide whether and when the transient has finished. The time lapse covered by $x_{w}$ is $\Delta t$ and $x_{w}$ is centered in the observation instant $t_{o b s}$. $\Delta t$ has been selected as the period of the lowest frequency tone measurable in the band assessed. The basic algorithm written as pseudocode is presented subsequently.

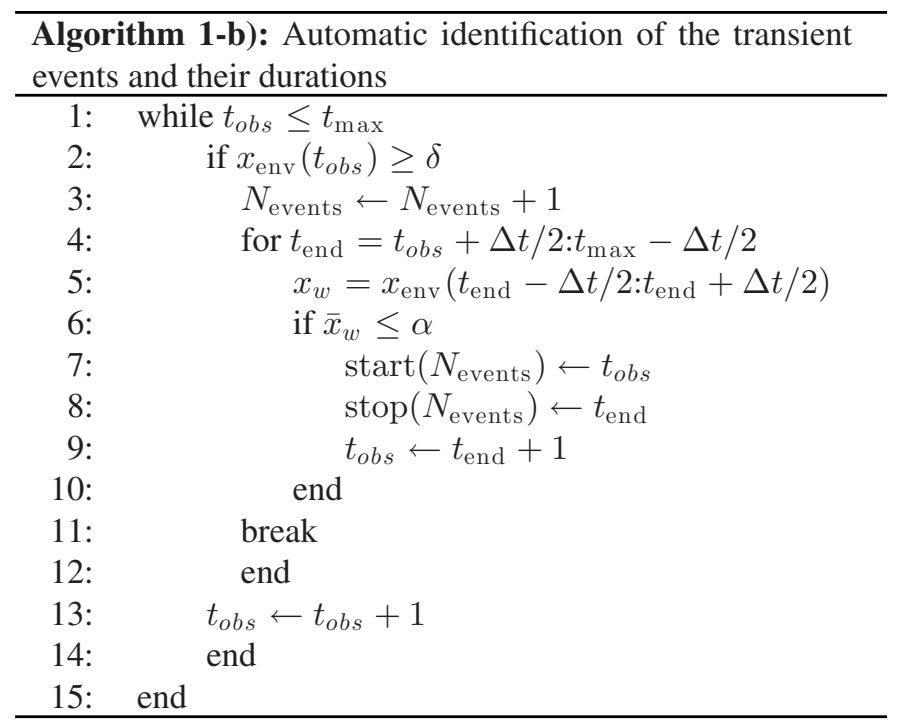

In consequence, the algorithm above uses $\delta$ to decide if during a certain instant $t_{o b s}$ a transient event has been encountered and then examines the mean value of $x_{w}$ to decide whether or not the transient event has finished in terms of a specified sensitivity factor $\alpha$. Then, the transient mode is the part of the EMI that occurs between the detected start and stop instants. The continuous EMI is calculated by subtracting the transient mode from the EMI signal.

Afterward, the duration of the $N_{\text {events }}$ detected transients are calculated as the difference between the vector containing the start time and the one containing the stop instants. The duration vector is statistically analyzed in order to find significant differences between the transient durations and also to reject outliers, 
particularly using the modified Thompson's Tau method [23]. Finally, the average transient event duration $\overline{\Delta t}_{\text {dur }}$ is calculated. The average transient event duration is an important parameter because it can be used to configure the proper record length when performing segmented memory acquisitions in order to optimize the exploitation of the available memory in oscilloscopes [6].

\section{Estimation of the Pulse Repetition Frequency}

The final step of the TMD algorithm is the estimation of the pulse repetition frequency $\hat{f}_{\text {rep }}$. Estimating $\hat{f}_{\text {rep }}$ is not mandatory for decomposing the EMI in the time domain, but it can be helpful for identifying the source of the inference. In this regard $\hat{f}_{\text {rep }}$ shall be estimated considering the impact of the jitter in successive transient events and the variability in the duration of the detected transient pulses due to the randomness of the measured signal amplitude with respect to the fixed threshold.

Therefore, for estimating the pulse repetition frequency $\hat{f}_{\mathrm{rep}}$, it is required to measure the central tendency of the inverse of the time shift between the start instants and also between the stop instants of successive transient events $\Delta t_{\text {start }}$ and $\Delta t_{\text {stop }}$. It is important to notice that for estimating the pulse repetition frequency there shall be at least two transient events detected.

The procedure for calculating $\hat{f}_{\text {rep }}$ is described in the following part of the algorithm. $\Delta t_{\text {start }}$ and $\Delta t_{\text {stop }}$ are onedimensional vector variables and the multiplicative inverse operation done in the third step is performed element-by-element. In the same manner, the median() function calculates the statistical median of the concatenated vectors $\left(\Delta t_{\text {start }}\right)^{-1}$ and $\left(\Delta t_{\text {stop }}\right)^{-1}$.

\begin{tabular}{l}
\hline Algorithm 1-c): Median pulse repetition frequency \\
\hline $1: \Delta t_{\text {start }} \leftarrow \operatorname{start}\left(1: N_{\text {events }}-1\right)-\operatorname{start}\left(2: N_{\text {events }}\right)$ \\
2: $\quad \Delta t_{\text {stop }} \leftarrow \operatorname{stop}\left(1: N_{\text {events }}-1\right)-\operatorname{stop}\left(2: N_{\text {events }}\right)$ \\
3: $\hat{f}_{\text {rep }} \leftarrow$ median $\left(\left[\left(\Delta t_{\text {start }}\right)^{-1}\left(\Delta t_{\text {stop }}\right)^{-1}\right]\right)$ \\
\hline
\end{tabular}

\section{APPLICATION EXAMPLES}

In this section, examples of EMI decomposition in the time domain will be presented. First, a low-frequency EMI signal was emulated using a controlled test signal synthesized using MATLAB and an Agilent 81160A pulse function arbitrary noise generator. The second scenario corresponds to a radiated emissions test. Measurements were performed with a TDEMI measurement system based on a Tektronix DPO5104B oscilloscope [8]. The assessed bands are in accordance with the CISPR 22 standard for conducted and radiated emissions measurements, respectively.

Despite the specific measurement conditions used for the next application examples, such as frequency range, resolution bandwidth, sampling rate, etc., it is important to remark that the method described previously is neither restricted by them nor by the standard used as a reference.

\section{A. Decomposition of a Synthesized EMI}

Fig. 2 shows a 1.6-ms time record of the EMI under consideration. This signal has been synthetically designed to include

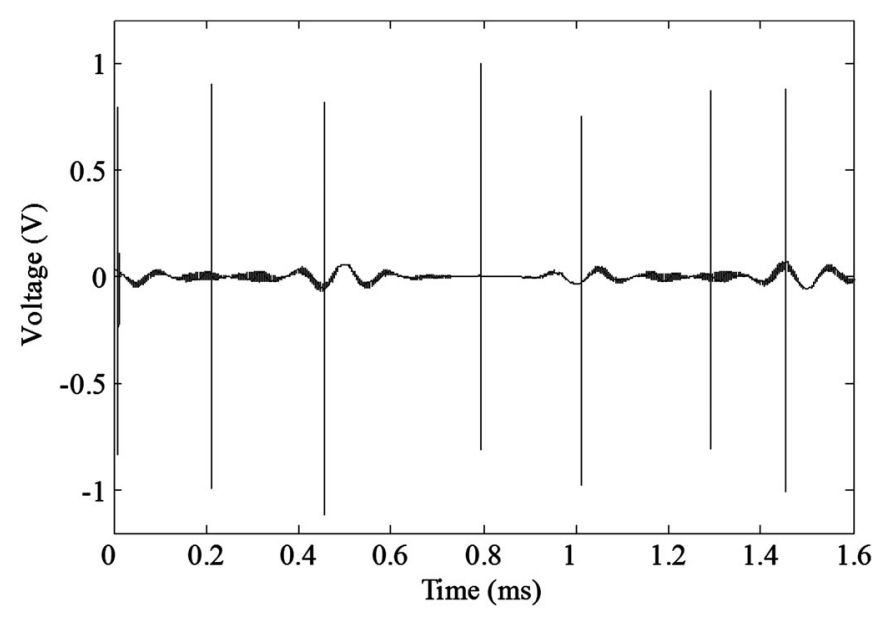

Fig. 2. Test signal for emulating an EMI measured in the time domain.

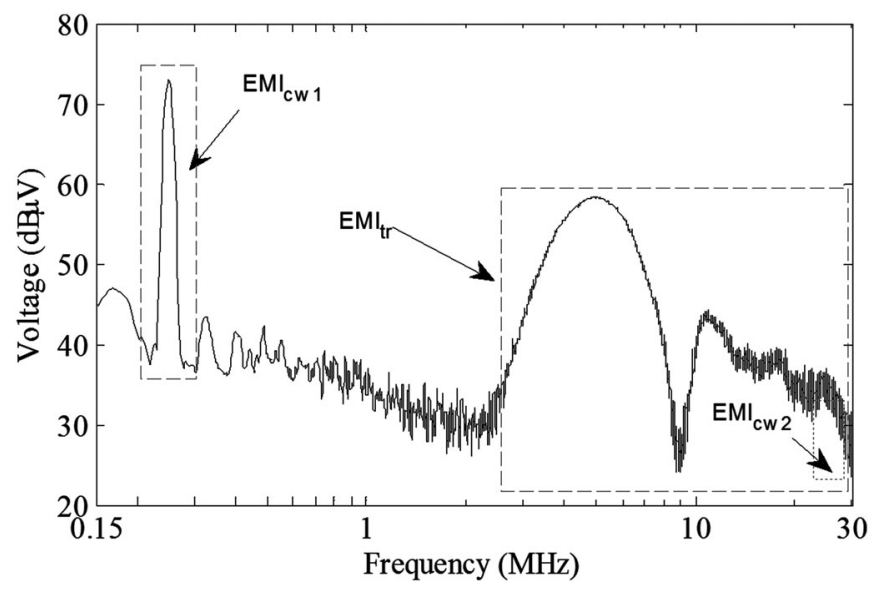

Fig. 3. EMI amplitude spectrum measured between $150 \mathrm{kHz}$ and $30 \mathrm{MHz}$.

transient/broadband and continuous wave/narrowband components. In particular, this example provides one quasi-periodic transient pulse with random jitter $\left(\mathrm{EMI}_{\mathrm{tr}}\right)$ and other three continuous wave signals. The continuous wave interferences are two amplitude modulated (AM) tones with carriers at $250 \mathrm{kHz}$ $\left(\mathrm{EMI}_{\mathrm{cw} 1}\right)$ and $27 \mathrm{MHz}\left(\mathrm{EMI}_{\mathrm{cw} 2}\right)$, respectively, and a very lowfrequency Gaussian modulated sinusoid pulse train $\left(\mathrm{EMI}_{\mathrm{cw} 3}\right)$.

The amplitude spectrum of the EMI under assessment is shown in Fig. 3. The spectral estimation has been calculated using the Welch's periodogram and the processing techniques explained in [4] and [6]. The narrow band signal $\mathrm{EMI}_{\mathrm{cw} 1}$ at approximately $250 \mathrm{kHz}$ corresponds to the AM broadcasting. In the upper part of the measured spectrum, the contribution of the broadband interference $\left(\mathrm{EMI}_{\mathrm{tr}}\right)$ overlaps the narrow band interference at $27 \mathrm{MHz}\left(\mathrm{EMI}_{\mathrm{cw} 2}\right)$ making it impossible to separate them using plain filtering. As expected, $\mathrm{EMI}_{\mathrm{cw} 3}$ is completely unnoticed in the amplitude spectrum shown in Fig. 3 because it is out of the measured frequency range.

In order to decompose the EMI in the time domain, first the transient pulses shall be detected and removed using the algorithms for TMD. The estimated pulse duration is $0.78 \mu \mathrm{s}$ and $\hat{f}_{\text {rep }} \approx 4.4 \mathrm{kHz}$. Fig. 4 shows the superposition of the seven 


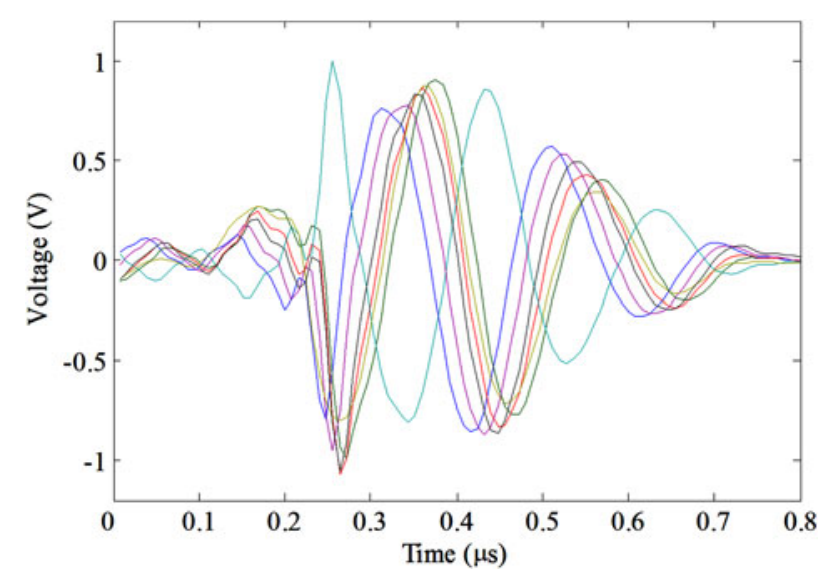

Fig. 4. Transient EMI pulses extracted from the EMI signal in time domain.

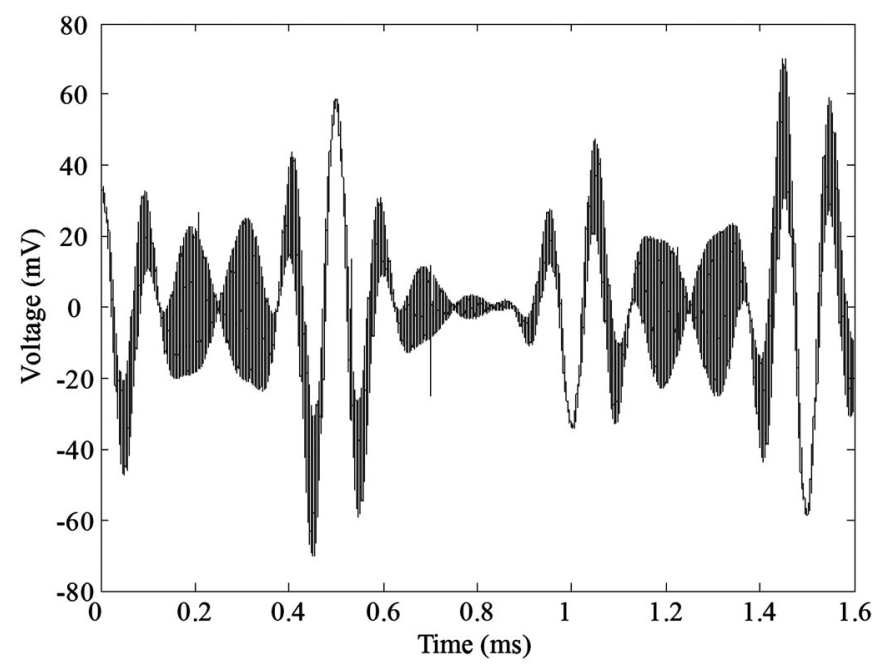

Fig. 5. Continuous wave part of the measured EMI after transient removal.

transient pulses identified in the recorded time frame of the assessed EMI. The amplitude of the transient EMI in the time domain is remarkably high in comparison with the continuous components. Fig. 4 verifies that the algorithm performs robustly even in the presence of jitter.

Once the transient pulses have been identified, they are removed from the EMI in order to perform the EMD on the continuous wave components of the EMI under assessment. Fig. 5 shows the EMI signal in the time domain after transient removal. Notice the amplitudes are under $80 \mathrm{mV}$, which is approximately two orders of magnitude below the transients.

By means of the EEMD, the continuous part of the EMI was decomposed into three IMF with simple waveforms: $\mathrm{IMF}_{1}$, $\mathrm{IMF}_{2}$, and $\mathrm{IMF}_{3}$. Fig. 6 presents the three IMF that comprise the assessed EMI. The residual is negligible because it is basically random noise introduced by the waveform generator. $\mathrm{IMF}_{1}$ and $\mathrm{IMF}_{3}$ are clearly AM signals, $\mathrm{EMI}_{\mathrm{cw} 1}$, and $\mathrm{EMI}_{\mathrm{cw} 2}$, respectively. $\mathrm{IMF}_{2}$ is the very low-frequency Gaussian-modulated sinusoid pulse train, which is $\mathrm{EMI}_{\mathrm{cw} 3}$. Even if $\mathrm{EMI}_{\mathrm{cw} 3}$ does not have frequency components in the $150 \mathrm{kHz}-30 \mathrm{MHz}$ band it is
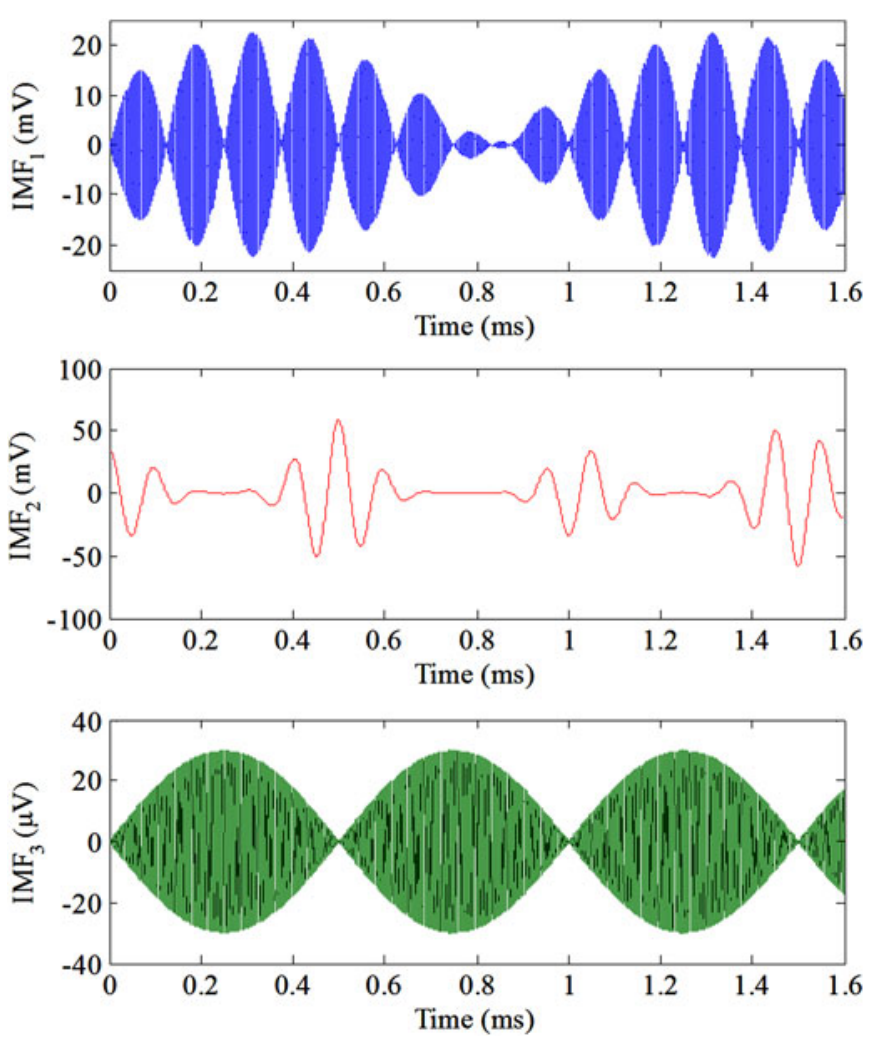

Fig. 6. IMFs of the continuous wave EMI.

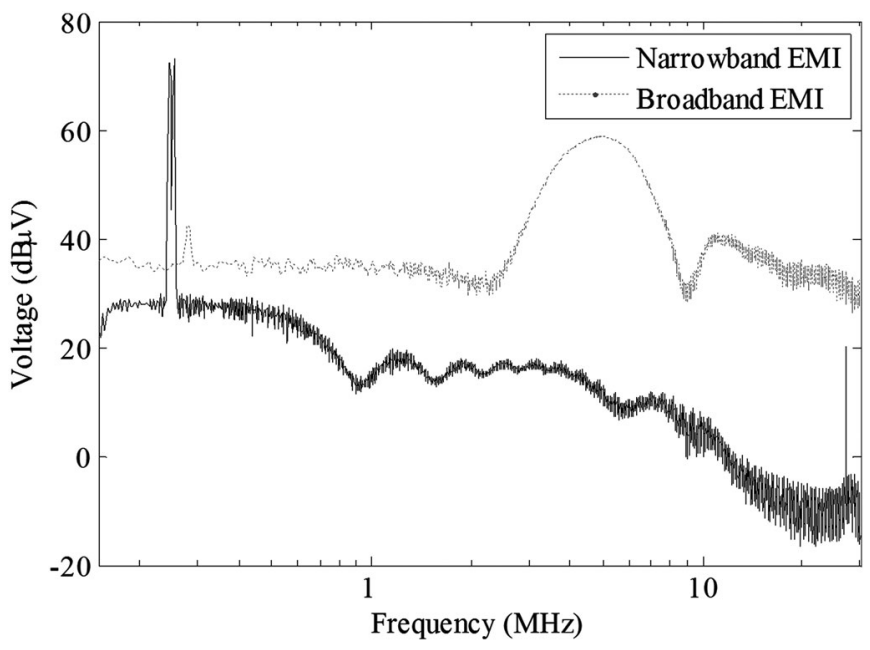

Fig. 7. Spectrum of the narrowband and broadband modes after the decomposition of the EMI measured with a TDEMI system.

a measurable part of the EMI since the time-domain approach provides the complete information of the signal within the instrument bandwidth.

Then, the IMF can be used to improve the results from the spectral estimations in TDEMI measurement systems. The key idea is to obtain the spectral contribution of each IMF using the optimum setting according to the type of signal [6], as shown in Fig. 7. Then, the total amplitude spectrum can be estimated superposing the contribution of each IMF [24]. 


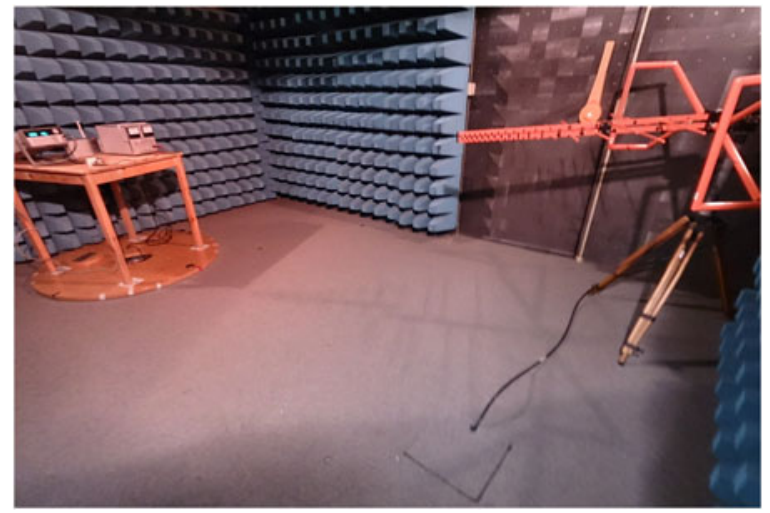

Fig. 8. Measurement setup.

Once the EMI signal is decomposed in the time domain, the frequency-domain results can be presented in terms of the broadband and narrowband IMF as shown in Fig. 8. After decomposition, both narrowband interferences are identified since the citizen band signal is no longer masked by the broadband spectral components of the transient pulse.

The denoising effect provided by the EMI decomposition is remarkable. Also, separating the broadband EMI from the narrowband interferences allow the algorithms to apply specific windowing and filtering; thus, improving the overall results through the reduction of the variability in the amplitude spectrum estimates.

\section{B. Decomposition of Radiated EMI}

Inside a fully anechoic chamber (FAC), two sources of radiated disturbances were placed acting as the EUT. The first EMI source is a circuit that generates radiated transients. The other noise source is a GSM jamming device in the $900-\mathrm{MHz}$ band. The door of the FAC was intentionally left open in order to capture noise from radio broadcasting. Radiated emissions measurements were performed in the $30 \mathrm{MHz}-1 \mathrm{GHz}$ band. The antenna was positioned for a 3-m EUT-to-test-antenna distance. A Schaffner CBL6143 bilog antenna in horizontal polarization was used. The test setup is shown in Fig. 8.

Emissions measurements were made with a TDEMI measurement system and, for validation purposes, with an EMI test receiver. On the one hand, the TDEMI measurement system used a digital oscilloscope Tektronix DPO5104B, a sampling rate of $5 \mathrm{GS} / \mathrm{s}$ and a record length of $1 \mathrm{~ms}$. On the other hand, frequency sweep measurements were done with a R\&S ESPI test receiver and the dwell time used was $1 \mathrm{~ms}$. In both cases, the resolution bandwidth was set to $120 \mathrm{kHz}$ in accordance with the CISPR specifications for bands $\mathrm{C}$ and $\mathrm{D}$. The overall measurement results are presented in Fig 9.

Fig. 9 indicates there is a broadband disturbance interfering the FM broadcasting band. Likewise, the emissions from the jammer interfere between 900 and $950 \mathrm{MHz}$ with GSM. The results obtained using both measurement systems (TDEMI and EMI test receiver) are in good agreement, that is, they provide the same information in terms of spectral estimation.

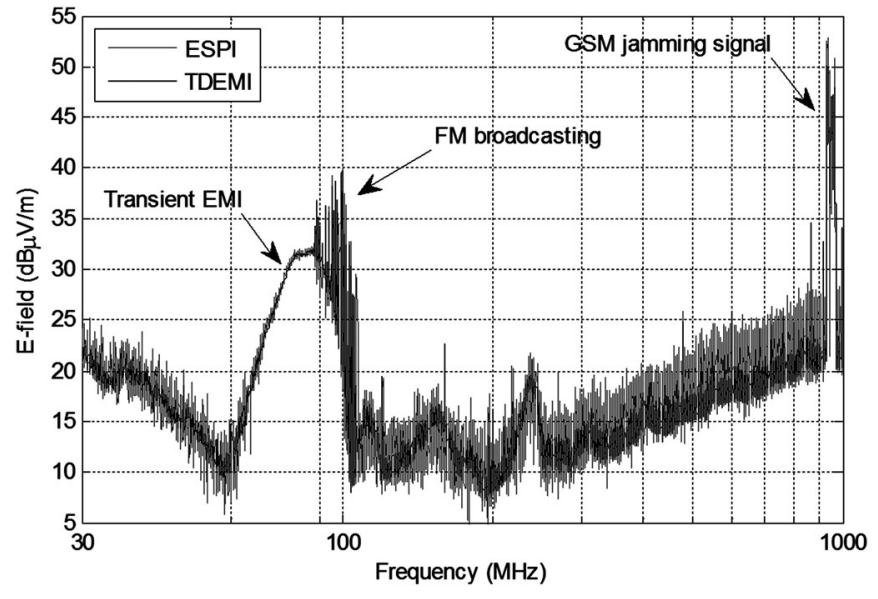

Fig. 9. Radiated EMI spectrum measured between $30 \mathrm{MHz}$ and $1 \mathrm{GHz}$.

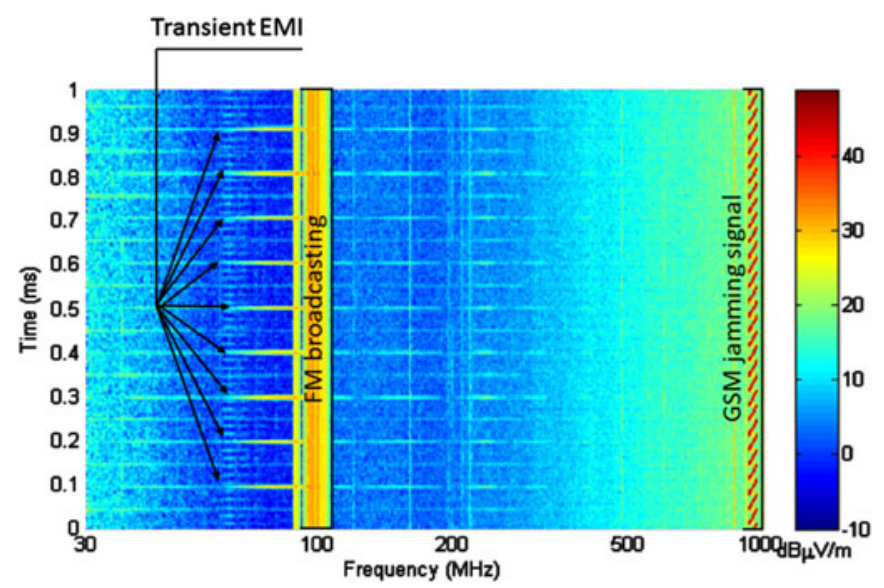

Fig. 10. Spectrogram of the measured EMI.

Fig. 10 shows the spectrogram of the EMI. The timefrequency representation of the measured disturbance verifies the transient behavior of the broadband interference. Fig. 10 also shows the GSM jamming signal is a linear chirp with a $31.25 \mu$ s period.

From Fig. 10, it is seen how the transient mode of the measured EMI overlaps the frequency spectrum of FM broadcasting. Furthermore, this spectrogram representation cannot be used for further signal decomposition since the transient duration is shorter than the length of the window required for the selected resolution bandwidth, that is, a resolution constraint in this type of time-frequency representation.

Nonetheless, through the decomposition algorithms presented in Sections II and III, the TDEMI measurement system is able to represent the radiated interferences as IMF. Fig. 11 shows the results of EMI decomposition process. The first IMF and $\mathrm{IMF}_{1}$ correspond to a transient signal with $10-\mathrm{kHz}$ pulse repetition frequency and $0.1-\mu$ s duration. $\mathrm{IMF}_{2}$ is the GSM jamming interference. Finally, $\mathrm{IMF}_{3}$ is the $\mathrm{FM}$ radio broadcasting signal.

The results exhibit some level of mode-mixing between $\mathrm{IMF}_{1}$ and $\mathrm{IMF}_{2}$. In the spectrum of $\mathrm{IMF}_{1}$ there is an attenuated 

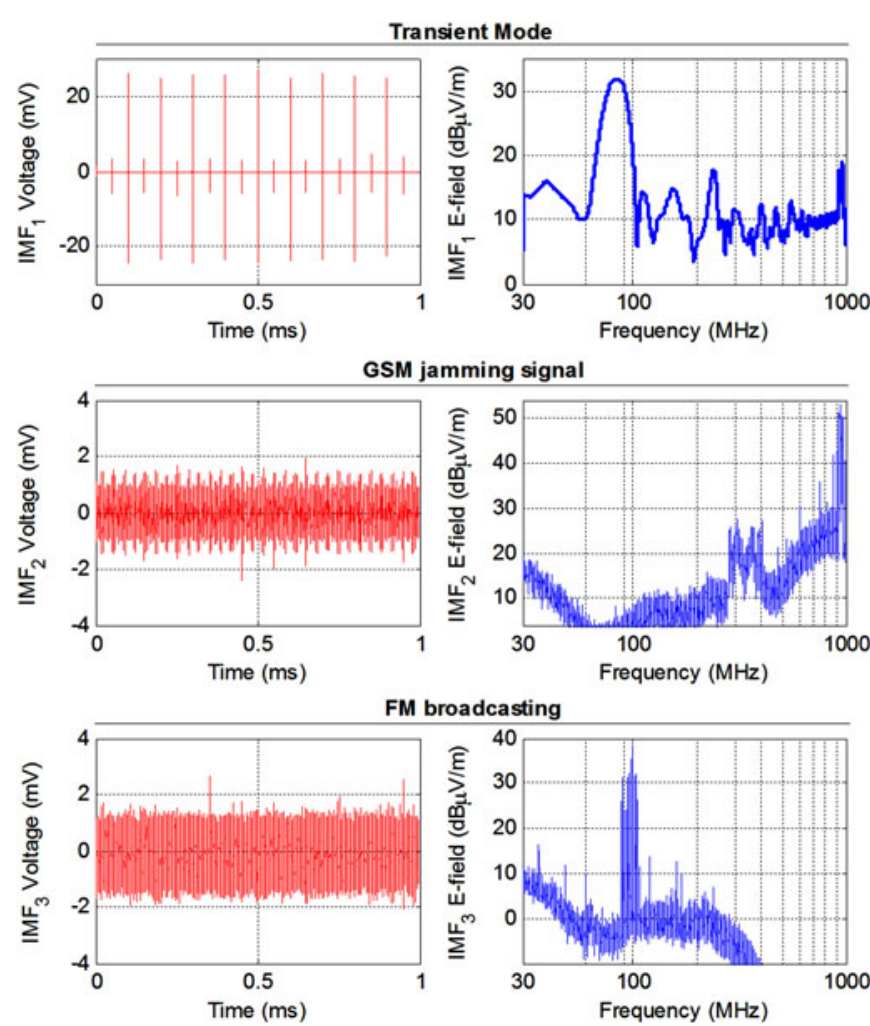

Fig. 11. IMFs in the time domain and in their spectral estimation.

component of the GSM jamming signal. Likewise, in the $\mathrm{IMF}_{2}$ there is a broadband component of noise that corresponds to the transient EMI. Nonetheless, the magnitude of the mode mixed components is near the noise floor, several decibels below the frequencies of maximum emissions. In this example, it would be possible to filter the mode mixed components since they do not overlap in frequency with the predominant interference of each IMF. Conversely, there was no mode-mixing between $\mathrm{IMF}_{3}$ and the other modes. This means that the transient mode was completely separated of the FM broadcasting signal, as shown in the spectrum of $\mathrm{IMF}_{1}$.

Aimed at validating the results, frequency sweep measurements were made only for the transient EMI, turning OFF the GSM jammer and closing the door of the anechoic chamber. Fig. 12 shows that the sensitivity of the TDEMI measurement system was improved by the noise reduction in the spectral estimation of the transient IMF.

The results confirm the decomposition algorithms were successful in identifying a finite set of IMF that describes accurately the main components of the EMI. EMI decomposition algorithms required a single time-domain acquisition and no prior assumption was imposed on the frequency content of the measured interference.

\section{DISCUSSION}

The decomposition of EMIs in the time domain is a methodology useful for analyzing complex disturbances. The combination of EMD and algorithms for transient mode separation

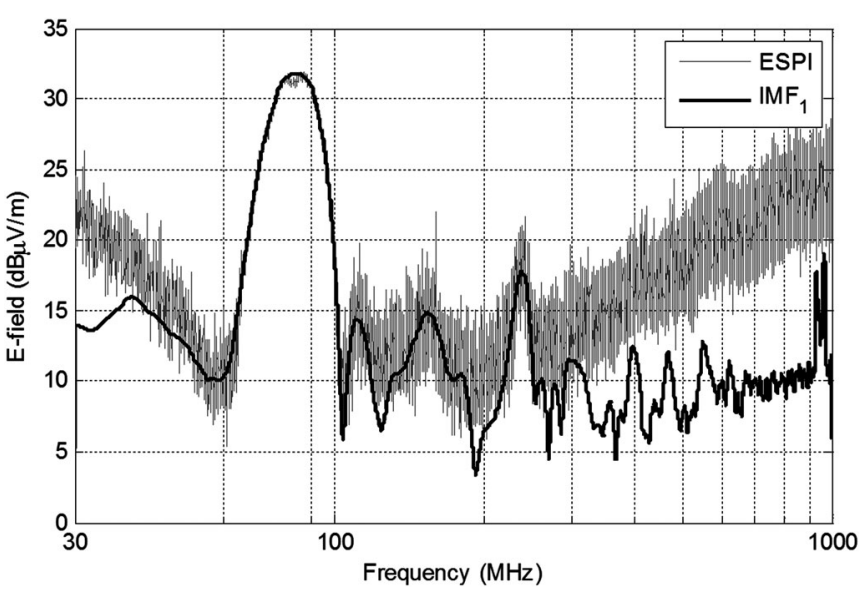

Fig. 12. Comparison between the amplitude spectrum of $\mathrm{IMF}_{1}$ and the measurement results of the radiated transient disturbances carried out with an EMI test receiver.

provide a heuristic approach to EMI analysis that allows the identification of a finite set of relatively simple IMF that describes the main components of the measured EMI signal.

In comparison with other signal decomposition approaches, the presented techniques have several advantages: it introduces neither distortion nor delay on the IMF, it requires no prior information on the spectral content of the measured EMI, it does not require a domain transformation, it provides IMFs that usually have a clear physical meaning, it is not constrained by time-frequency resolution limits, and it allows a straightforward implementation through software.

Therefore, the decomposition of EMI in the time domain provides several new practical applications to be explored in TDEMI measurement systems, especially for those based on general-purpose oscilloscopes. For example, the EMI decomposition in the time domain could be used for finding, tracking, and identifying sources of an EMI in complex environments, that is, inside large machinery and installations. Also, EMI decomposition in the time domain is potentially applicable for the cancellation of unwanted background ambient noise in emissions measurements performed in situ, as required in industrial environments or when assessing interferences of railway systems.

However, EMI decomposition in the time domain has some drawbacks, and its performance is improvable. In particular, with the EMD algorithm, it is not possible to know beforehand how many IMF will be identified. Therefore, the required time to process the signal is not bounded, and this could be impractical for emissions testing measurements purposes. A possibility to reduce the processing time is to establish a fixed number of intrinsic modes obtainable on the basis of the analysis of a preliminary measure.

\section{CONCLUSION}

This paper presented the customization of the EMD with transient separation capabilities for the application of EMI decomposition in the time domain. The algorithms are capable of 
identifying a finite set of IMFs that describe accurately the main components of the EMI. The presented algorithms required a single time-domain acquisition to perform the EMI decomposition and no prior assumption was made on the frequency content of the measured interferences. The result of the decomposition process provides insights of the measurement results through the use of specific time-domain signal processing techniques.

The main advantage of the applying EMI decomposition in the time domain is the capability of studying the contribution of each IMF to the overall EMI. This allows identifying different narrowband, broadband, and ambient noise components within measurement results. That knowledge would allow TDEMI measurement systems applying specific digital processing techniques for improving measurement results by means of selective denoising.

\section{REFERENCES}

[1] Information Technology Equipment—Radio Disturbance Characteristics -Limits and Methods of Measurement, CISPR Standard EN 55022, 2008.

[2] M. Pous and F. Silva, "Full-Spectrum APD measurement of transient interferences in time domain," IEEE Trans. Electromagn. Compat., vol. 56, no. 6, pp. 1352-1360, Dec. 2014.

[3] G. Costa, M. Pous, A. Atienza, and F. Silva, "Time-domain electromagnetic interference measurement system for intermittent disturbances," in Proc. Int. Symp. Electromagn. Compat., 2014, pp. 833-837.

[4] M. A. Azpúrua, M. Pous, and F. Silva, "A measurement system for radiated transient electromagnetic interference based on general purpose instruments," in Proc. Int. Symp. Electromagn. Compat., 2015, pp. 11891194.

[5] P. M. Oliveira and V. Barroso, "Uncertainty in the time-frequency plane," in Proc. IEEE 10th Workshop Statist. Signal Array Process., 2000, pp. 607-611.

[6] M. A. Azpúrua, M. Pous, S. Çakir, M. Çetintaş, and F. Silva, "Improving time-domain EMI measurements through digital signal processing," IEEE Electromagn. Compat. Mag., vol. 4, no. 2, pp. 82-91, Apr.-Jun. 2015.

[7] E. X. Alban, M. E. Magana, H. G. Skinner, and K. P. Slattery, "Statistical modeling of the interference noise generated by computing platforms," IEEE Trans. Electromagn. Compat., vol. 54, no. 3. pp. 574-584, Jun. 2012.

[8] R. Yang, B. Zhang, D. Qiu, and Z. Liu, "Time-;frequency and wavelet transforms of EMI dynamic spectrum in chaotic converter," IEEE Trans. Power Electron., vol. 24, no. 4. pp. 1083-1092, Apr. 2009.

[9] M. Pous and F. Silva, "Prediction of the impact of transient disturbances in real-time digital wireless communication systems," IEEE Electromagn. Compat. Mag., vol. 3, no. 3. pp. 76-83, Jul./Sep. 2014.

[10] M. A. Azpurua, M. Pous, and F. Silva, "On the statistical properties of the peak detection for time-domain EMI measurements,' IEEE Trans. Electromagn. Compat., vol. 57, no. 6, pp. 1374-1381, Dec. 2015.

[11] Y.-Y. Hong and Y.-Q. Bao, "FPGA implementation for real-time empirical mode decomposition," IEEE Trans. Instrum. Meas., vol. 61, no. 12, pp. 110, Dec. 2012

[12] N. E. Huang, Z. Shen, S. R. Long, M. C. Wu, H. H. Shih, Q. Zheng, N.-C. Yen, C. C. Tung, H. H. Liu, N. E. Huang, Z. Shen, S. Long, M. Wu, H Shih, Q. Zheng, N.-C. Yen, C. Tung, and H. Liu, "The empirical mode decomposition and the Hilbert spectrum for nonlinear and non-stationary time series analysis," Proc. Roy. Soc. London. Ser. A, Math. Phys. Eng. Sci., vol. 454, no. 1971, pp. 903-995, 1998.

[13] M. Blanco-Velasco, B. Weng, and K. E. Barner, "ECG signal denoising and baseline wander correction based on the empirical mode decomposition," Comput. Biol. Med., vol. 38, no. 1, pp. 1-13, 2008.

[14] N. Bi, Q. Sun, D. Huang, Z. Yang, and J. Huang, "Robust image watermarking based on multiband wavelets and empirical mode decomposition.", IEEE Trans. Image Process., vol. 16, no. 8, pp. 1956-1966, Aug. 2007.

[15] T. Tanaka and D. P. Mandic, "Complex empirical mode decomposition," IEEE Signal Process. Lett., vol. 14, no. 2, pp. 101-107, Feb. 2007.

[16] A. Eftekhar, C. Toumazou, and E. Drakakis, "Empirical mode decomposition: Real-time implementation and applications," J. Signal Process. Syst., vol. 73, no. 1, pp. 43-58, 2013.
[17] Y. Gao, G. Ge, Z. Sheng, and E. Sang, "Analysis and solution to the mode mixing phenomenon in EMD," in Proc. 1st Int. Congr. Image Signal Process., 2008, vol. 5, pp. 223-227.

[18] P. Flandrin, G. Rilling, and P. Goncalves, "Empirical mode decomposition as a filter bank," IEEE Signal Process. Lett., vol. 11, no. 2, pp. 112-114, Feb. 2004.

[19] P. J. J. Luukko, J. Helske, and E. Räsänen, "Introducing libeemd: A program package for performing the ensemble empirical mode decomposition," Comput. Statist., pp. 1-13, 2015, early eccess.

[20] K. Dragomiretskiy and D. Zosso, "Variational mode decomposition," IEEE Trans. Signal Process., vol. 62, no. 3, pp. 531-544, Feb. 2014.

[21] T. O'Haver. (2015). "A pragmatic introduction to signal processing with applications in scientific measurement," [Online]. Available: https://terpconnect.umd.edu/ toh/spectrum/TOC.html

[22] C. B. Moler, Numerical Computing With MATLAB, 1st ed. Philadelphia, PA, USA: SIAM, 2004.

[23] R. Willink, Measurement Uncertainty and Probability. Cambridge, U.K.: Cambridge Univ. Press, 2013.

[24] C. Keller and K. Feser, "Fast emission measurement in time domain," IEEE Trans. Electromagn. Compat., vol. 49, no. 4, pp. 816-824, Nov. 2007.

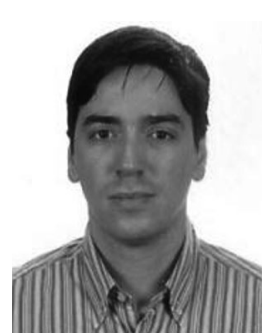

Marco A. Azpúrua (M'13) received the B.Sc. degree in telecommunications engineering, in 2008, and the M.Sc. degree in electrical engineering from the Universidad Central de Venezuela, Caracas, Venezuela, in 2013. He is currently working toward the Ph.D. degree at the Electromagnetic Compatibility Group, Universitat Politècnica de Catalunya, Barcelona, Spain.

He was a Researcher in the Applied Electromagnetics Laboratory, Instituto de Ingeniería (FII), Caracas. He is a Member of the Venezuelan Standardization Committee for the Telecommunication Sector of CODELECTRA and FODENORCA and has participated in the adoption of the local standards of electromagnetic compatibility. His research interests include electromagnetic compatibility, antenna and microwave measurement technologies, estimation of measurement uncertainty in complex systems, and validation methods.

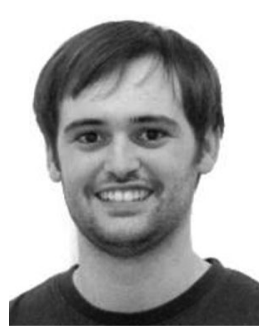

Marc Pous was born in Barcelona, Spain, in 1983. He received the M.Sc. degree in telecommunications engineering and the Ph.D. degree in radiated transient interferences and digital communication systems evaluation from the Universitat Politècnica de Catalunya, Barcelona, Spain, in 2009 and 2015, respectively.

From 2003 to 2006, he was with the LGAI Technological Centre, Department of Electromagnetic Compatibility. In 2006, he was with the Electromagnetic Compatibility Group, Universitat Politècnica de Catalunya, where he has been participating in international and national research projects related with automotive, aerospace, railway and medical industries. His research interests include development of time domain measurement techniques to capture interferences which are not properly measured following the harmonized EMC standards.

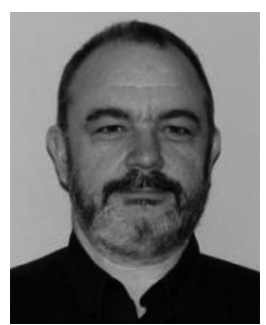

Ferran Silva (S'93-M'98) received the M.Sc. and $\mathrm{Ph} . \mathrm{D}$. degrees from the Universitat Politècnica de Catalunya (UPC), Barcelona, Spain, in 1989 and 1997, respectively.

Since 2000, he has been the Director of the Electromagnetic Compatibility Group, Universitat Politècnica de Catalunya (GCEM-UPC) performing technology transfer activities to the industrial sector. $\mathrm{He}$ is currently an Associate Professor of Electronics with the Department of Electronic Engineering, UPC. His research interests include electromagnetic compatibility (EMC) in near field and time domain, including transients, with application to automotive, medical systems and installations. He has made more than 90 publications in journals and conferences and contributed to the Wiley Encyclopedia of Biomedical Engineering. He has participated in 14 research projects related to EMC.

Dr. Silva is a Member of the IEEE EMC Society and the Head of the Spanish chapter of the same society; he is also Member of the Spanish Standardization Committees SCTC77-210 and the CTN208 SCCISPR210A. He was the Chairman of the EMC Europe 2006 International Symposium. Since then, he belongs to the Board of Chairmen of EMC Europe. 